Kardiologe 2010 • 4:77-77

DOI 10.1007/s12181-010-0259-1

(c) Springer-Verlag 2010

\author{
F.-W. Mohr \\ Klinik für Herzchirurgie, Leipzig
}

\title{
Kardiovaskuläre Medizin - eine gemeinsame Aufgabe
}

Veränderungen des Myokards, sowie Perfusionsstörungen oder -defezite exakt beschreiben und auch quantifizieren, so dass insbesondere bei den Patienten mit grenzwertig revaskulisierbaren Myokardischämien eine exakte Erfolgsaussage vor der Intervention erfolgen kann. Dies wiederum sollte dann zur endgültigen Therapieentscheidung wesentlich beitragen und in jedem Fall solchen Entscheidungen vorausgehen.

Die Häufigkeit degenerativer Herzklappenerkrankungen ist ansteigend und die herzchirurgischen Abteilungen sind mit der Zunahme an alten „Klappenpatienten“ mit multiplen Comorbiditäten konfrontiert. Sehr erfolgreich konnte in den vergangenen Jahren das chirurgische Trauma durch minmal invasive Operationsmethoden und verbesserte Anästhesieverfahren (fast track) reduziert werden, welch dann zu anhaltend guten Ergebnissen auch bei diesen Patienten führen.

Moderne sehr exakte Bildgebung wie z. B. Ultraschall und MRT erlauben uns heute präoperativ die spezifische Klappenmorphologie in Zusammenhang mit der Ventrikelpathologie exakt bildlich in der präoperativen Diagnostik zu beschreiben und darzustellen. Das was der Chirurg wissen muss kann heute präopertiv „digital“ erfasst und dreidimensional dargestellt werden! Jetzt liegt es an den Chirurgen sich dessen zu bedienen, seine Therapieverfahren voraussagbar darauf einzustellen und evtl. auch den am besten geeigeten Chirurgen dafür auszuwählen. Dieses Vorgehen hat z.B. dazu geführt, dass die Rekonstruktionsrate bei Mitralklappeninsuffizienz in Deutschland mit einer über 66\%igen Erfolgsrate weltweit führend ist. Dieser Fakt ist überwiegend den minimal invasiven Opera- tionsmethoden (Erfolgsrate $>80 \%$ ) geschuldet.

\section{Neue Operationsverfahren}

Manche Operationsverfahren oder auch Interventionen schienen vor 15 Jahren noch unmöglich, manchmal jedoch führt die entschlossene Pionierarbeit Einzelner und die nachfolgende enge Zusammenarbeit von Kardiologen, Herzchirurgen und Ingenieuren zu „Neuen Methoden“: Ich denke PCI und katheterbasierter Aortenklappenersatz sind beide herausragende Beispiele und eng mit dem Namen von Grüntzig und Cribier verbunden.

Es ist atemberaubend zu beobachten, wie in kürzester Zeit in den vergangenen fünf Jahren der transfemorale und transapikale Aortenklappenersatz vorangetrieben wurden. Verbunden mit der technischen Entwicklung von modernen Hybrid-OP-Sälen und der engen Zusammenarbeit von Herzchirurgen, Kardiologen, Anästhesisten und Industriepartnern.

Diese Themen werden uns in Mannheim beschäftigen, gemeinsam müssen wir lernen „unser Herz“ besser zu verstehen und dann auch gemeinsam die richtigen Therapiemaßnahmen einleiten, die ohne Zweifel durch die moderne Bildgebung kritisch zu steuern und zu überwachen sind.

Ihr

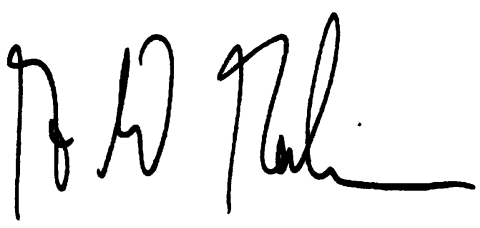

Prof. Dr. Friedrich-Wilhelm Mohr
Multi Slice CT und 2-3D-Echokardiographie können heute die morphologischen 